\title{
Percepción De Estrés Y Prácticas Parentales En Estudiantes De Psicología De Nuevo Ingreso
}

\author{
José Esael Pineda Sánchez \\ Rosa Mariela González Hernández \\ Angélica Romero Palencia \\ Rebeca Guzmán-Saldaña
}

Universidad Autónoma del Estado de Hidalgo. Instituto de Ciencias de la

Salud, Área Académica de Psicología, Laboratorio de Psicofisiología

doi: 10.19044/esj.2017.v13n17p325 URL:http://dx.doi.org/10.19044/esj.2017.v13n17p325

\begin{abstract}
The entrance to the university life causes changes and demands that generate high stress and difficulties for the adaptation. There is evidence that parental overinvolvement, specifically psychological control (induction of guilt or disqualification), causes problems in the psychosocial adjustment of children and adolescents. Few studies address the impact of parental practices on the adaptive processes of young adults. The aim of the present study was to describe, compare by sexes, and associate the perception of stress and prevailing parental practices among college admission students of psychology career. A non-experimental transectional, comparativecorrelational study was performed 127 students (70.08\% female, 29.92\% male) with an average age of 19 from a public university in Hidalgo, Mexico participated. The Scale of Parental Practices for Adolescents (Andrade \& Betancourt, 2008) and the Stress Perception Scale (González \& Landero, 2007) were applied. No differences were found between men and women in the perception of stress, but in parental practices of maternal communication, maternal knowledge about son activities and induction of guilt, devaluation and excessive maternal criticism were found differences. Likewise, correlations between stress and the dimensions of maternal-filial respect, maternal imposition, induction of guilt, devaluation and excessive criticism, paternal-filial respect and paternal imposition on women were found; and between paternal and filial stress and respect in males. It is suggested that the role of parents in child rearing has a greater impact on the adaptive behavior of women in university life.
\end{abstract}

Keywords: Parental practices, stress, colleges, parental overinvolvement, parenting styles 


\section{Resumen}

El ingreso a la vida universitaria provoca cambios y demandas que generan estrés elevado y dificultades para la adaptación. Existe evidencia de que el sobreinvolucramiento parental, específicamente el control psicológico (inducción de culpa o descalificación), provoca problemas en el ajuste psicosocial de los niños y adolescentes. El objetivo del presente estudio fue describir, comparar por sexo, y asociar la percepción del estrés y las prácticas parentales predominantes en universitarios de nuevo ingreso a la licenciatura en psicología. Se utilizó un diseño no experimental transeccional, comparativo-correlacional. Participaron 127 estudiantes (70.08\% mujeres; 29.92\% hombres) con una edad promedio de 19 años de una universidad pública de Hidalgo, México. Se aplicó la Escala de Prácticas Parentales para Adolescentes (Andrade \& Betancourt, 2008) y la Escala de Percepción del Estrés (González \& Landero, 2007). No se encontraron diferencias entre hombres y mujeres en la percepción de estrés, pero sí en las prácticas parentales, específicamente en comunicación materna, conocimiento materno sobre actividades del hijo (a) e inducción de culpa, devaluación y críticas excesivas maternas. Asimismo, se encontraron correlaciones entre estrés psicosocial y las dimensiones de respeto maternofilial, imposición materna, inducción de culpa, devaluación y críticas excesivas, respeto paterno-filial e imposición paterna en las mujeres; y entre estrés y respeto paterno-filial en los varones. Se sugiere que el papel de ambos padres en la crianza de los hijos tiene mayor impacto sobre la conducta adaptativa a la vida universitaria en las mujeres.

Palabras clave: Prácticas parentales, estrés psicosocial, universitarios, sobreinvolucramiento parental, estilos de crianza.

\section{Introducción}

El ingreso a la Universidad representa para el estudiante una etapa de transición que puede provocar problemas de adaptación al nuevo contexto relacionados con separaciones, establecimiento de nuevas relaciones interpersonales y académicas, administración del tiempo, economía, el desempeño académico, etc. Por ello, el nivel de estrés en los estudiantes durante el primer año de la licenciatura es elevado (Ion, 2013; Medrano, Galleano, Galera, \& del Valle Fernández, 2010). Se estima que cerca del $25 \%$ de los estudiantes que ingresan a la Universidad abandonan sus estudios durante el primer año, por lo que este período es fundamental para predecir el ajuste a la vida universitaria y la trayectoria académica (D’Lima, Winsler, \& Kitsantas, 2014). Se calcula que la eficiencia terminal (número de alumnos que ingresan y concluyen con éxito un nivel educativo) en las 
instituciones de nivel superior de México oscila entre el 39\% y 73\%. Sin embargo, este cálculo se realiza sin considerar la trayectoria escolar, refiriéndonos con ello a los comportamientos de los estudiantes asociados a elementos de aprobación o reprobación y el desempeño académico a lo largo de un ciclo escolar (García \& Barrón, 2011), situación que dificulta establecer la tasa de deserción, es decir, el abandono de las actividades escolares antes de un ciclo escolar y los factores psicosociales relacionados con este fenómeno (López, Albíter, \& Ramírez, 2008; Mares et. al, 2013; Cuéllar, 2014).

El ajuste a la vida universitaria es un fenómeno complejo que requiere del estudiante la implementación de estrategias de afrontamiento que contribuyan al proceso adaptativo eficiente (Perea \& Mcllveen, 2014), es decir, un adecuado afrontamiento ayuda al estudiante al desarrollo académico de un semestre en particular así como de la vida universitaria en general así como en el ajuste social, personal, emocional y sirve como preámbulo para continuar en el semestre; un afrontamiento activo como la reevaluación positiva y la solución de problemas se relaciona con mayores niveles de bienestar psicológico que un afrontamiento centrado en la evitación (Abdullah, Elias, Uli, \& Mahyuddin, 2010). De acuerdo con Gallander y Yaffe (2000) existen tres modelos que podrían explicar el ajuste del alumno a la vida universitaria, el primero se relaciona con el manejo de las emociones, la competencia y el desarrollo de la autonomía; el segundo enfoque, enfatiza los elementos psicosociales previos tales como el estatus económico y el género, que juegan un papel importante en el desgaste; y finalmente, el tercero propone que el modelo parental de socialización y las relaciones paterno-filiales impactan en el desarrollo universitario.

La familia es uno de los principales soportes en la transición hacia la vida universitaria y las prácticas de crianza pueden tener un efecto sobre el ajuste a ella, facilitando u obstaculizando el afrontamiento al estrés y el bienestar de los estudiantes (Mattanah, Ayers, Brand, \& Brooks, 2010; Lewis, West, Roberts, \& Noden, 2015; Schwanz, Palm, Hill-Chaoman, \& Broughton, 2014; Smojver-Ažić, Dorčić, \& Juretić, 2015). La sociedad cambia la forma en cómo las familias y sus miembros adoptan roles de crianza de acuerdo al género, por ejemplo, algunas familias son más tradicionales y otras utilizan actitudes democráticas. Las prácticas de crianza varían de un padre a otro y tienen efectos diferentes en los hijos. Desde la perspectiva social los padres se conciben como instructores y modelos de las actitudes hacia los roles de género de sus hijos. Por ejemplo, ambos padres comunican sus creencias respecto al género de acuerdo a actividades sociales estereotipadas, reforzando actitudes sexuales típicas (Marks, Lam , \& McHale, 2009). Las actitudes de los padres hacia las hijas se tornan hacia el desarrollo de habilidades afectivas, actitudes femeninas y conductas 
relacionadas con el hogar, ejerciendo una mayor presión y control sobre ellas. Por otra parte, en los varones se promueve más la autonomía, ya que culturalmente los hombres son estereotipados como proveedores del hogar y más vinculados a tareas laborales fuera del hogar (Galet \& Alzás, 2014).

Respecto a la crianza existen dos modelos de análisis del impacto parental sobre la conducta de los hijos. El primero se centra en los estilos de crianza (autoritario, democrático, permisivo y negligente), y se relaciona con el clima emocional que se genera a través de las relaciones paterno-filiales. El segundo corresponde al enfoque dimensional que considera la influencia de las conductas específicas de la crianza (prácticas parentales) sobre los hijos, tales como: comunicación, autonomía, control psicológico, control conductual e imposición (Darling \& Steinberg, 1993; Jiménez-Iglesias \& Moreno, 2015).

Ahora bien, las prácticas parentales que fomentan el afecto y el apoyo permiten al individuo desarrollar las herramientas necesarias para desenvolverse efectivamente en nuevos contextos, incluidos los académicos (Shook, Forehand, Jones, Dorsey, \& Brody, 2010). Contrariamente, un menor apoyo parental provoca más estrés y problemas emocionales (Rueger, Malecki, \& Demaray, 2010) . En este contexto se entiende al estrés como la respuesta que se da cuando las demandas son evaluadas como excesivas por el sujeto y sus recursos no son suficientes para enfrentarlas, desencadenando reacciones conductuales, fisiológicas y psicológicas (Fink, 2010). En investigaciones realizadas con universitarios, se reporta que mayor apoyo social sirve como protector para los efectos de estrés (Cohen, Kamarck, \& Mermelstein, 1983; Friedlander, Reid, Shupak, \& Cribbie, 2007; Crockett, Iturbide, Torres, McGinley, Raffaelli, \& Carlo, 2007) y para el ajuste universitario (Ramsay, Jones, \& Barker, 2007) y en general a la salud mental (Moeini, Shafii, Hidarnia, Reza, Birashk, \& Allahverdipour, 2008).

Diversas investigaciones sugieren que las prácticas de crianza negativas, como el sobreinvolucramiento parental, provocan insatisfacción con la vida familiar y un bienestar psicológico disminuido en los niños y adolescentes. Por otra parte, la presencia del apoyo materno se relaciona con actitudes de mayor competencia social y cognitiva en los hijos; en cambio, la ausencia de este apoyo se relaciona con un desajuste social (p. e. Shook et al., 2010). Respecto a la figura paterna, algunos modelos teóricos en psicología sugieren que el padre no se ve como promotor del desarrollo y estructuración de personalidad del hijo, a comparación de la madre, que, por el vínculo y el apego que genera con el niño, se considera como una figura más significativa en la vida de los individuos (Quaglia \& Castro, 2007). Es así que, en cuanto a los estilos paternales, aunque la mayoría de los casos los progenitores tienen estilos similares, se ha visto que si la madre es más indiferente o más autoritaria tiene consecuencias perjudiciales en 
comparación cuando el padre es más controlador (Marks et al., 2009). En los estudiantes universitarios existen pocos estudios sobre las prácticas parentales y su relación con el afrontamiento al estrés (Capano, González, \& Massonnier, 2016; Conley, Travers \& Bryant, 2015). La mayoría de las investigaciones realizadas se centran en infantes y adolescentes (Betancourt, 2007; Blondal \& Adalbjarnardottir, 2009; Lee, Beckert, Wu, \& Kuan, 2011; Martínez, García, \& Aguirre-Acevedo, 2015) con relación a otras variables como depresión (Andrade, Betancourt, Vallejo, Celis \& Rojas, 2012), problemas externalizados e internalizados (Betancourt, 2007; Espejel, 2015), ansiedad (Franco, Pérez, \& de Dios, 2014), autoestima (Alonso \& Román, 2014). Asimismo se han relacionado con la crianza en general y no con prácticas parentales específicas (Martínez \& García, 2011).

Por lo anterior, el objetivo fundamental de la presente investigación fue conocer la relación de las prácticas parentales y la percepción del estrés en estudiantes de universitarios de nuevo ingreso. Para ello, se describen los niveles de estrés presentados por los estudiantes y las diferencias por sexo con respecto de esta percepción, se reportan las prácticas parentales predominantes en esta población y se describen las diferencias por sexo en dichas prácticas. Por último, se describen las relaciones entre la percepción del estrés y las prácticas parentales en universitarios de nuevo ingreso a la licenciatura en psicología.

\section{Método}

\section{Participantes}

Se trabajó con una muestra intencional compuesta por 127 estudiantes universitarios de nuevo ingreso (29.9\% hombres y $70.1 \%$ mujeres) con una media de edad de 19 años, de la licenciatura de psicología de una universidad del estado de Hidalgo, México.

\section{Diseño}

Se realizó un estudio no experimental, de diseño transeccionalcorrelacional.

\section{Instrumentos} mexicana:

Se aplicaron dos escalas validadas y confiabilizadas para población

Escala de Prácticas Parentales para Adolescentes (PP-A) de Andrade y Betancourt (2008). Instrumento autoaplicable de 80 reactivos (40 para cada padre), con cuatro opciones de respuesta tipo Likert: Nunca, Pocas veces, Muchas veces y Siempre, que reporta la percepción que tienen los hijos de las conductas de sus padres hacia ellos. Clasifica las prácticas parentales en cinco dimensiones maternas: Comunicación $(\alpha=0.92)$, Autonomía $(\alpha=$ 
0.86), Control Conductual ( $\alpha=0.84)$, Imposición $(\alpha=0.80)$ y Control Psicológico $(\alpha=0.80)$; y cuatro paternas: Comunicación y Control Conductual $(\alpha=0.97)$, Autonomía $(\alpha=0.94)$, Imposición $(\alpha=0.90)$ y Control Psicológico $(\alpha=0.90)$.

Escala de Percepción del Estrés (PPS) de González y Landero (2007). Instrumento autoaplicable de 14 reactivos con un formato de cinco respuestas tipo Likert, donde 0 es nunca, 1 casi nunca, 2 de vez en cuando, 3 a menudo, 4 muy a menudo; que mide la manera en que los jóvenes perciben el estrés psicológico. Se clasifican los resultados de acuerdo a los datos normativos para población mexicana de acuerdo al género y la edad $(\leq 24$ años; $\square=23.20 ; \sigma=7.25$ ). A partir del valor promedio reportado se debe sumar y restar el valor de la desviación estándar, el resultado es el rango de estrés esperable, por debajo de este valor es estrés bajo y por arriba estrés alto (González-Ramírez, Rodríguez-Ayán, \& Landero, 2013).

\section{Procedimiento}

Se procedió a obtener la firma del consentimiento informado por medio de una plática informativa en la que se explicaba la finalidad de la investigación y se enfatizó la participación voluntaria por parte de los estudiantes. Se aplicaron las pruebas a la población blanco en las instalaciones de una universidad del estado de Hidalgo, México. Posteriormente se procedió a dar por terminada la evaluación y se explicó a los estudiantes que los resultados de sus pruebas estarían disponibles para ellos en el transcurso del curso. Después de calificar las pruebas se descartaron las pruebas que tuvieran más del $20 \%$ de las escalas sin contestar. Finalmente se procedió a realizar el análisis de los datos.

\section{Resultados y análisis de datos}

Para estimar los niveles de percepción del estrés se utilizaron los parámetros para población mexicana propuestos por González-Ramírez, Rodríguez-Ayán y Landero (2013). Se encontró que 28.57 \% de la población reportó estrés bajo, $65.87 \%$ moderado y $5.56 \%$ alto.

Se procedió a realizar una comparación por sexo de los niveles de estrés. Dada la diferencia entre el tamaño de las muestras en hombres y mujeres, se obtuvo una muestra aleatoria de mujeres del 50\% con la finalidad de asegurar la homogeneidad. Se observa una tendencia mayor en los varones en los niveles de estrés percibido (Tabla 1).

Tabla 1 Porcentajes del nivel de estrés percibido en hombres y mujeres.

\begin{tabular}{ccc}
\hline Nivel de Estrés Percibido & $\begin{array}{c}\text { Mujeres } \\
\mathrm{N}=50\end{array}$ & $\begin{array}{c}\text { Hombres } \\
\mathrm{N}=38\end{array}$ \\
\hline Bajo & $30.00 \%$ & $21.62 \%$ \\
Regular & $68.00 \%$ & $70.27 \%$ \\
Alto & $02.00 \%$ & $08.11 \%$ \\
\hline
\end{tabular}


Posteriormente se realizó una prueba t de Student para muestras independientes utilizando de la muestra segmentada los puntajes crudos y no se observaron diferencias estadísticamente significativas $(t=-.951 ; p$ $=.344)$.

Asimismo, se encontraron diferencias significativas entre hombres y mujeres en las prácticas maternas, específicamente en las dimensiones de comunicación, control conductual y control psicológico con tendencia hacia las prácticas percibidas por las mujeres (Tabla 2).

Tabla 2 Diferencias en las prácticas parentales en hombres y mujeres.

\begin{tabular}{ccccc}
\hline $\begin{array}{c}\text { Prácticas } \\
\text { Parentales } \\
\text { Maternas }\end{array}$ & $\begin{array}{c}\text { Sig. } \\
\text { (bilateral) }\end{array}$ & $\mathbf{t}$ & $\begin{array}{c}\text { Mujeres } \\
\square(\boldsymbol{\sigma})\end{array}$ & $\begin{array}{c}\text { Hombres } \\
\square(\boldsymbol{\sigma})\end{array}$ \\
\hline $\begin{array}{c}\text { Comunicación } \\
\text { Control }\end{array}$ & .014 & 2.509 & $3.06(0.75)$ & $2.65(0.77)$ \\
$\begin{array}{c}\text { Conductual } \\
\text { Control }\end{array}$ & .001 & 4.363 & $3.30(0.63)$ & $2.62(0.75)$ \\
Psicológico & .046 & -2.030 & $1.40(0.53)$ & $1.62(0.50)$ \\
\hline
\end{tabular}

Se realizó un análisis de correlación por medio del índice productomomento de Pearson, para la muestra total y se observaron correlaciones positivas entre la percepción del estrés y prácticas maternas de imposición y control psicológico. De igual forma, se observaron correlaciones negativas entre estrés y prácticas maternas de comunicación y autonomía; y entre estrés y prácticas paternas de autonomía (ver tabla 4).

Posteriormente, se realizó un análisis de correlación por medio de la prueba de Spearman entre estrés percibido y prácticas parentales por sexo, debido al número de sujetos de la muestra. Se encontraron correlaciones negativas entre estrés y prácticas de autonomía paterna en los varones (ver tabla 3).

Por otra parte, en las mujeres se observaron correlaciones negativas entre estrés y prácticas de autonomía materna y paterna, y correlaciones positivas entre estrés y prácticas de control psicológico materno e imposición de ambos padres (ver tabla 3).

Tabla 3 Correlación entre el Estrés Percibido y las Prácticas Parentales en hombres y mujeres.

\begin{tabular}{lccc}
\hline & $\begin{array}{c}\text { Estrés Percibido } \\
\text { Global } \\
\mathrm{N}=127\end{array}$ & $\begin{array}{c}\text { Estrés Percibido } \\
\text { Hombres } \\
\mathrm{N}=38 \\
\text { Correlación Pearson } \\
\text { Sprelación }\end{array}$ & $\begin{array}{c}\text { Estrés Percibido } \\
\text { Mujeres } \\
\mathrm{N}=50\end{array}$ \\
$\begin{array}{l}\text { Correlación } \\
\text { Spearman }\end{array}$ \\
\hline $\begin{array}{l}\text { Comunicación: entre la madre } \\
\text { yel adolescente. }\end{array}$ & $\mathbf{- . 1 8 6}^{*}$ & .048 & -.202 \\
$\begin{array}{l}\text { Autonomía: El respeto que } \\
\text { muestra la madre en las } \\
\text { decisiones que toma su hijo. }\end{array}$ & $-\mathbf{- . 2 7 8}^{* *}$ & -.160 & $-.317^{* *}$ \\
$\begin{array}{l}\text { Imposición: El grado en que la } \\
\text { madre impone sus creencias y }\end{array}$ & $\mathbf{. 3 5 4}^{* *}$ & & \\
\end{tabular}


conductas al hijo.

Control Psicológico: Inducción de culpa, devaluación y críticas excesivas maternas al adolescente.

Autonomía: El respeto que $-.191^{*}$ $-.357^{*}$ $-.242^{*}$ muestra el padre en las decisiones que toma su hijo.

Imposición: El grado en que el padre impone sus creencias $\mathrm{y}$ conductas al hijo.

\footnotetext{
${ }^{*}$ La correlación es significativa en el nivel 0,05 (bilateral).

${ }^{* *}$ La correlación es significativa en el nivel 0,01 (bilateral).
}

\section{Discusión}

El objetivo primordial de la presente investigación fue conocer la importancia que tienen las prácticas parentales y la percepción del estrés en los estudiantes de nuevo ingreso a la carrera de psicología. En primer lugar, se encontró que en general los estudiantes de nuevo ingreso reportan un nivel moderado de estrés, lo que coincide con lo reportado por Ancer, Meza, Pompa, Torres y Landero (2011) quienes estudiaron la percepción del estrés en una población similar. Sin embargo, existen otros estudios que se han enfocado en estudiar el nivel de estrés académico en los alumnos, durante el primer año de la carrera universitaria, que reportan niveles elevados de estrés (Domínguez, Guerrero, \& Domínguez, 2015; García-Ros, Pérez-González, Pérez-Blasco, \& Natividad, 2012;), causando un desajuste en el funcionamiento mental y físico del estudiante (Rayle \& Chung, 2007). Este contraste sugiere que existen diferencias importantes entre el estrés concebido como un constructo relacionado con los aspectos generales de la vida y el estrés relacionado con los aspectos exclusivamente académicos. No obstante, es importante resaltar que los intervalos de evaluación de estos constructos con respecto del inicio de los cursos universitarios han sido diferentes en las investigaciones. Esta disparidad en el período que se realiza la evaluación podría explicar algunas de las diferencias entre los distintos constructos, por lo que se sugiere que en investigaciones futuras se considere este aspecto como una variable de control.

En cuanto a las diferencias entre sexos en la percepción del estrés, diversas investigaciones realizadas en población mexicana de estudiantes universitarios de múltiples carreras, utilizando la escala PPS (González \& Landero, 2007) muestran resultados distintos. Por ejemplo, Leiner y Jiménez (2011) trabajaron con una muestra de 2454 estudiantes universitarios de diversas carreras y encontraron diferencias significativas, con puntuaciones mayores en las mujeres que en los varones. Por otra parte, en estudios realizados en estudiantes universitarios de la carrera de psicología exclusivamente, no se reportan diferencias significativas entre sexos con 
referencia al nivel de estrés (González \& Landero, 2008). En el presente estudio no se encontraron diferencias estadísticamente significativas en la percepción del estrés entre hombres y mujeres. Esto puede sugerir que el tipo de carrera afecta la percepción del estrés con respecto del sexo, sin embargo, no debe olvidarse que la cantidad de hombres en la carrera de psicología es significativamente menor con respecto de otras carreras.

Con relación a las prácticas parentales, el presente estudio buscó evaluar si la percepción de las prácticas parentales tiene alguna relación sobre los adultos jóvenes de recién ingreso a la universidad, debido a que la mayoría de estudios que evalúan este constructo han trabajado con niños y adolescentes. Por ejemplo, Betancourt y Andrade (2011) encontraron diferencias entre las prácticas de control reportadas por hombres y mujeres adolescentes, con una tendencia en las mujeres a percibir mayor nivel de control. Asimismo un factor predictor para los problemas de conducta en los adolescentes, es el control ejercido por la madre, resaltando el papel preponderante que tiene en la crianza. De la misma manera, la percepción que tienen hijos e hijas sobre la crianza del padre y la madre da mayor peso a las prácticas maternas, principalmente en cuanto a control y comunicación (Capano, et al., 2016; Ruvalcaba-Romero, Gallejos-Guajardo, Caballo, \& Villegas-Guinea, 2016;). Los resultados encontrados en nuestra investigación concuerdan con lo reportado en población adolescente, sugiriendo que el impacto de las prácticas parentales sobre los individuos puede tener continuidad aún en la vida adulta. Lo anterior contrasta con lo que reportan otros investigadores (Rodríguez, Del Barrio \& Carrasco, 2009; JiménezIglesias \& Moreno, 2015) quienes argumentan que a medida que los hijos crecen, muestran una disminución en la percepción de métodos disciplinarios parentales. Sin embargo, nuestros datos sugieren que la crianza es fundamental en edades tempranas, pero sigue jugando un papel importante en la vida adulta y en su adaptación al medio ambiente.

Las correlaciones negativas encontradas entre las prácticas parentales de autonomía y comunicación, y la percepción del estrés en la población estudiada, indican que el sobreinvolucramiento de los padres en las decisiones de los hijos es uno de los principales generadores de estrés (Lewis,et al., 2014; Segrin, Givertz, Swaitkowski, \& Montgomery, 2015). Asimismo, las correlaciones positivas entre las prácticas de control y el estrés ratifican esta tendencia (Gallander \& Yaffe, 2000). La relación entre las variables de estudio se acentúa más en el grupo femenino, revelando que en las mujeres el impacto de las prácticas parentales tiene mayor preponderancia como generador de estrés. En este sentido nuestros resultados coinciden con los de otras investigaciones (Andrade, et al., 2012), que además suponen una relación entre las prácticas parentales maternas de control psicológico y la sintomatología depresiva en mujeres. Esto denota 
que los estilos parentales de la madre tienden a percibirse como coercitivos y controladores en relación con los estilos paternos.

Finalmente, los varones reportan mayor nivel de estrés, con respecto de las mujeres, en su relación con el padre solamente en aspectos relacionados al respeto que este tiene en cuanto a las decisiones tomadas por el hijo. Para Marks et al., (2009) los padres se ven más involucrados en actividades tipificadas por sexo con hijos varones que las madres. De esta forma, los padres que promocionan más la autonomía, generan en sus hijos una mejor calidad de vida (Capano et al., 2016). Estos datos ratifican la importancia de la familia en nuestra cultura y el peso que se le da a la crianza estricta y a los roles de género (Gill-Rivas, Greenberger, Chen, \& Montero, 2003). Es decir, los datos de la presente investigación refuerzan la hipótesis de que las prácticas parentales maternas tienen un impacto mayor sobre el comportamiento de los hijos, y ejercen una percepción de mayor control psicológico sobre las mujeres.

La principal aportación de la presente investigación se vincula a la importancia que tienen las prácticas parentales en los procesos de adaptación en la adultez temprana, específicamente la que se relaciona a los contextos universitarios. La relación entre las prácticas parentales y los niveles de estrés contribuye a la comprensión de los patrones conductuales que deben considerarse al momento de abordar a esta población, con el objetivo de apoyarles en su proceso de adaptación a la vida universitaria. Finalmente, el explorar el impacto de las prácticas parentales específicas, en lugar de aspectos generales como los estilos parentales, sobre la vida adulta y su relación con los procesos adaptativos del ser humano a los diferentes ámbitos de socialización, promueve la implementación de programas psicológicos de atención que ponderen la contribución de dichas prácticas a la conformación de la personalidad y las estrategias de ajuste a la vida social de los individuos.

Entre las principales limitaciones del presente estudio, se puede mencionar que existen escasos instrumentos que evalúen las prácticas parentales en la edad adulta, por lo que se utilizó una prueba cuyo rango máximo de edad es de 18 años. Sin embargo, la similitud en los resultados con las investigaciones predecesoras nos lleva a considerar que este aspecto se comporta de igual manera con la población de jóvenes adultos. Se propone que las futuras investigaciones consideren la comparación entre constructos de estrés académico y psicosocial, además de observar si el período dentro del ciclo escolar en que se realiza la evaluación tiene un impacto diferencial sobre estas variables. Por último, se debe contemplar este tipo de investigaciones con estudiantes de diferentes profesiones con el objeto de definir si existen diferencias de acuerdo al perfil de la carrera elegida. 


\section{References:}

1. Abdullah, M. C., Elias, H., Uli, J., \& Mahyuddin, R. (2010). Relationship between Coping and University Adjustment and Academic Achievement amongst First Year Undergraduates in a Malaysian Public University. International Journal of Arts and Sciences, 3(11), 379-392.

2. Alonso, J., \& Román, J. M. (2014). Nivel sociocultural, prácticas educativas familiares y autoestima de los hijos en edades tempranas. Revista de Investigación Educativa, 32(1), 187-202.

3. Ancer, L., Meza, C., Pompa, E. G., Torres, F., \& Landero, R. (2011). Relación entre los niveles de autoestima y estrés en estudiantes universitarios. Enseñanza e Investigación en Psicología, 16(1), 91101.

4. Andrade, P., Betancourt, D., Vallejo, A., Celis B. S., \& Rojas, R. M. (2012). Prácticas parentales y sintomatología depresiva en adolescentes. Salud Mental, 35(1), 29-36.

5. Andrade, P., \& Betancourt, D. (2008). Prácticas parentales: Una medición integral. En S. Rivera-Aragón, R. Diaz-Loving, R. Sánchez-Aragón, e I. Reyes-Lagunes. La Psicología Social en México. Volumen XII. México: AMEPSO, 561-565.

6. Betancourt, D., \& Andrade, P. (2011). Control Parental y Problemas Emocionales y de Conducta en Adolescentes. Revista Colombiana de Psicología, 20(1), 27-41.

7. Betancourt, D. (2007). Control parental y problemas internalizados y externalizados en niños y adolescentes (Tesis de Doctorado). Universidad Nacional Autónoma de México, México.

8. Blondal, K. S., \& Adalbjarnardottir, S. (2009). Parenting Practices and School Dropout: A Longitudinal Study. Adolescence, 44(176), 729-749.

9. Capano, A., González, M. D. L., \& Massonnier, N. (2016). Estilos relacionales parentales: estudio con adolescentes y sus padres. Revista de Psicología, 34(2), 413-444.

10. Cohen, S., Kamarck, T., \& Mermelstein, R. (1983). A global measure of perceived stress. Journal of Health and Social Behavior, 24(4), 385-396.

11. Conley, C. S., Travers, L. V., \& Bryant, F. B. (2015). Promoting Psychosocial Adjustment and Stress Management if First-Year College Students: The Benefits of Engagement in a Psychosocial Wellness Seminar. Journal of American College Health, 61(2), 7586. DOI:10.1080/07448481.2012.754757 
12. Crockett, L. J., Iturbide, M. I., Torres, R. A., McGinley, M., Raffaelli, M., \& Carlo, G. (2007). Acculturative Stress, Social Support, and Coping: Relations to Psychological Adjustment among Mexican American College Students. Cultural Diversity and Ethnic Minority Psychology, 13(4), 347-355.

13. Cuéllar, D. (2014). Deserción escolar en educación media superior: análisis de los factores escolares para la toma de decisiones de política pública (Tesis de Maestría). Facultad Latinoamericana de Ciencias Sociales, México.

14. Darling, N., \& Steinberg, L. (1993). Parenting style as context: an interactive model. Psychological Bulletin, 113(3), 487-496.

15. D’Lima, G. M., Winsler, A., \& Kitsantas, A. (2014). Ethnic and Gender Differences in First-Year College Students' Goal Orientation, Self-Efficacy, and Extrinsic and Intrinsic Motivation. The Journal of Educational Research, 345-356. DOI:10.1080/00220671.2013.823366

16. Domínguez, R. A., Guerrero, G. J., \& Domínguez, J. G. (2015). Influencia del estrés en el rendimiento académico de un grupo de estudiantes universitarios. Educación y ciencia, 4(43), 31-40.

17. Espejel, S. D. (2015). Prácticas parentales y problemas externalizados en niños (Tesis de Licenciatura). Universidad Nacional Autónoma de México, México.

18. Fink, G. (2010). Stress: Definition and History. En Stress Science Neuroendocrinology (pp. 3-9). Oxford: Elsevier Inc

19. Franco, N. N, Pérez, N. M. A., \& de Dios, P. M. J. (2014). Relación entre los estilos de crianza parental y el desarrollo de ansiedad y conductas disruptivas en niños de 3 a 6 años. Revista de Psicología Clínica con niños y adolescentes, 1(2), 149-156.

20. Friedlander, L. J., Reid, G. J., Shupak, N., \& Cribbie, R. (2007). Social Support, Self-Esteem, and Stress as Predictors of Adjustment to Universitary Among First-Year Undergraduates. Journal of Collage Students Development, 48(3), 259-274.

21. Galet, C., \& Alzás, T. (2014). Trascendencia del rol de género en le educación familiar. Cuerpo Abierto, 33(22), 97-114.

22. Gallander, M., \& Yaffe, M. (2000). First-Year Student's Adjustment to Universitary Life as a Functions of Relationship with Parents. Journal of Adolescent Research, 15(1), 9-37.

23. García, R., \& Barrón, C. (2011). Un estudio sobre la trayectoria escolar de los estudiantes de doctorado en Pedagogía. Perfiles Educativos, 33(131), 94-113.

24. García-Ros, R., Pérez-González, F., Pérez-Blasco, J., \& Natividad, L. A. (2012). Evaluación del estrés académico en estudiantes de nueva 
incorporación a la universidad. Revista Latinoamericana de Psicología, 44(2), 143-154.

25. Gill-Rivas, V., Greenberger, E., Chen C., \& Montero, M. (2003). Understanding depressed mood in the context of a family-oriented culture. Adolescence, 38(149), 93-109.

26. González, M. T., \& Landero, R. (2007). Factor Structure of the Perceive Stress Scale (PSS) in a sample from Mexico. The Spanish Journal of Psychology, 10(1), 199-206.

27. González, M. T., \& Landero, R. (2008). Síntomas psicosomáticos y estrés: comparación de un modelo estructural entre hombre y mujeres. Ciencia UANL, 11(4), 403-410.

28. González-Ramírez, M. T., Rodríguez-Ayán, M. N., \& Landero, R. (2013). The Perceived Stress Scale (PSS): Normative Data and Factor Structure for Large-Scale sample from Mexico. The Spanish Journal of Psychology, 16(e47), 1-9.

29. Hernández, C. M. (2015). Prácticas parentales y su relación con la adaptación en la adolescencia (Tesis de Licenciatura). Universidad Nacional Autónoma de México, México.

30. Ion, A. (2013). Adaptation and stress for the first year university students. Procedia Social and Behavioral Sciences, 78, 718-722.

31. Jiménez-Iglesias, A., \& Moreno, C. (2015). La influencia de las diferencias entre el padre y la madre sobre el ajuste adolescente. Anales de Psicología, 31(1), 367-377.

32. Lee, C. T., Beckert, T. E., Wu, C. I., \& Kuan, P. Y. (2011). The Impact of Marital Discord of Parents on Taiwanese Adolescents' Academic Achievement: The Mediating and Moderating Effect of Maternal Parenting Practice. Journal of Comparative Family Studies, 827-850.

33. Leiner, M., \& Jiménez, P. (2011). Un estudio comparativo del estrés percibido en estudiantes de ciencias administrativas y biológicas en tiempo de violencia. Contaduría y Administración, 233, 99-119.

34. Lewis, J., West, A., Roberts, J., \& Noden, P. (2015). Parents' involvement and university students' independence. Families, Relationships and Societies: An International Journal of Research and Debate, 4(3), 417-432.

35. López, A., Albíter, A., \& Ramírez, L. (2008). Eficiencia terminal en la Educación Superior, la necesidad de un nuevo paradigma. Revista de la Educación Superior, 37(2), 135-151.

36. Mares, G., González, L. F., Rivas, O., Rocha, H., Rueda, E., Rojas, L. E., Cruz, D., \& López, R. (2013). Trayectorias discontinuas en educación superior: el caso de alumnos de la carrera de psicología de 
Iztacala, México. Revista Mexicana de Investigación en Psicología, 5(1), 71-81.

37. Marks, J., Lam, C. B., \& McHale, S. (2009). Family Patterns of Gender Role Attitudes. National Institute of Child Health and Human Development, 61(3-4), 221-234.

38. Martínez, M., \& García, M. C. (2011). Implicaciones de la crianza en la regulación del estrés. Revista Latinoamericana de Ciencias Sociales, Niñez y Juventud, 2(9), 535-545.

39. Martínez, M., García, M. C., \& Aguirre-Acevedo, D. C. (2015). Respuesta al estrés, temperamento y crianza en niños colombianos de un año. Revista Latinoamérica de Ciencias Sociales, Niñez y Juventud, 13(2), 1065-1080.

40. Mattanah, J. F., Ayers, J. F., Brand, B. L., \& Brooks, L. J. (2010). A Social Support Intervention to Ease the College Transition: Explorating Main Effects and Moderators. Journal of College Students Development, 51(1), 93-108.

41. Medrano, L. A., Galleano, C., Galera, M., \& del Valle, R. (2010). Creencias irracionales, rendimiento y deserción académica en ingresantes universitarios. Liberabit, 16(2), 183-192.

42. Moeini, B., Shafii, F., Hidarnia, A., Reza, G., Birashk, B., \& Allahverdipour, H. (2008). Perceived stress, self-efficacy and its relations to psychological well-being status in Irian male high school students. Social Behavior and Personality: An International Journal, 36, 257-266.

43. Ramírez, M. E. (2005). Padres y desarrollo de los hijos: prácticas de crianza. Estudios Pedagógicos, 31(2), 167-177.

44. Ramsay, S., Jones, E., \& Barker, M. (2007). Relationship between adjustment and support types: Young and mature-aged local and international first year university students. Higher Education, 54, 247-265.

45. Rayle, A. D., \& Chung, K. Y. (2007). Revisiting first-year college students' mattering: social support, academic stress, and the mattering experience. Journal College Student Retention, 9(1), 21-37.

46. Rodríguez, M. A., Del Barrio, M. V., \& Carrasco, M. A. (2009). ¿Cómo perciben los hijos la crianza materna y paterna? Escritos de Psicología, 2(2), 10-18.

47. Rueger, S. Y., Malecki, C. K., \& Demaray, M. K. (2010). Relationship between multiple sources of perceived social support and psychological and academic adjustment in early adolscence: Comparisions across gender. Journal of Youth and Adolescence, 39, 47-61. 
48. Ruvalcaba-Romero, N., Gallegos-Guajardo, J., Caballo M., V., \& Villegas-Guinea, D. (2016). Prácticas parentales e indicadores de salud mental en adolescentes. Revista Psicología desde el Caribe, 33(3), 223-236.

49. Perera, H., \& McIlveen, P. (2014). The role of Optimism and Engagement Coping in College Adaptation: a career construction model. Journal of Vocational Behavior, 84, 395-404.

50. Quaglia, R., \& Castro, F. V. (2007). El papel del padre en el desarrollo del niño. International Journal and Development and Educational Psychology, 2, 167-182.

51. Sánchez, J., \& Hidalgo, M. V. (2003). De las ideas de las madres a las interacciones con sus bebés. Anales de Psicología, 19(2), 279292.

52. Schiffrin, H., Liss, M., Miles-McLean, H., Geary, K., Earchull, M., \& Tashnner, T. (2014). Helping or hovering? The effects of helicopter parenting on college students' well-being. Journal Child Family Studies, 23, 548-557.

53. Schwanz, K. A., Palm, L. J., Hill-Chaoman, C. R., \& Broughton, S. F. (2014). College Students' Perceptions of Relationship with Parents and Performance. American Journal of Educational Research, 2(1), 13-17.

54. Segrin, C., Givertz, M., Swaitkowki, P., \& Montgomery, N. (2015). Overparenting is Associated with Child Problems and Critical Family Envirionment. Journal of Child and Family Studies, 24, 470-479.

55. Shook, S. E., Jones, D. J., Forehand, R., Dorsey, S., \& Brody, G. (2010). The mother-coparent relationship and youth adjustment: A study of African American single-mother families. Journal of Family Psychology, 24(3), 243-251.

56. Smojver-Ažić, S., Dorčić, T. M., \& Juretić, J. (2015). Contribution of parental attachment and involvement to the academic, emotional and social adjustment to college: A three-year longitudinal study. Psihološka obzorja: Horizons of psychology, 24, 21-32. 